\title{
MEDIUM FREQUENCY BURST EMISSIONS: A TERRESTRIAL ANALOG TO SOLAR TYPE III BURSTS?
}

\author{
J. LaBelle*
}

\begin{abstract}
Auroral Medium Frequency Burst (MFB) is the least understood of three types of terrestrial auroral emissions detectable at ground level. MFB consists of broadband $(500-2000 \mathrm{kHz})$ left-polarized impulsive emissions typically occurring for a few minutes at the onset of polar substorms, one of the most energetic phenomena in the terrestrial magnetosphere. Recent observations of the source location and fine structure of MFB provide the best opportunity yet to test theoretical models of the generation mechanism. Proposed mechanisms include mode conversion of Langmuir or electron cyclotron sound waves excited via resonant interactions with auroral electrons; the former has been shown under certain conditions to predict the frequency-time characteristics of MFB fine structure.
\end{abstract}

\section{Introduction}

Two broad classes of nonthermal planetary radio emissions can be identified: direct radiation mechanisms, such as the electron cyclotron maser mechanism, whereby unstable particle distributions directly generate electromagnetic (EM) radiation; and indirect mechanisms, such as plasma emission, whereby electrostatic waves or turbulence mediate the exchange of energy from the particles to the EM waves. The latter are sometimes referred to as mode conversion radiation because of the requirement to convert wave energy from electrostatic to escaping electromagnetic modes. Mode conversion radiation occurs in a wide range of space plasmas; examples include planetary continuum radiations, which result from conversion of upper hybrid waves, and many of the solar radio bursts which involve Langmuir waves. Much closer to Earth, some auroral radio emissions are of the mode conversion type [LaBelle and Treumann, 2002]. The best understood examples are

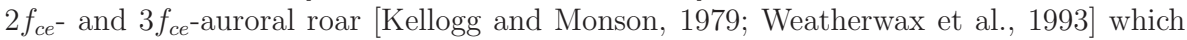
arise from conversion to LO-mode of upper hybrid waves generated near harmonics of the electron cyclotron frequency by cyclotron resonance with auroral electrons [Yoon et

* Department of Physics and Astronomy, Dartmouth College, Hanover, New Hampshire, USA 
al., 1996]. The mechanism is almost identical to that of planetary continuum radiations, which also arise from mode conversion of upper hybrid waves. However, because auroral roar emissions are observable at ground level and their source regions detectable with sounding rocket instrumentation [e.g., Samara et al., 2004], they are more accessible to experimental study than are planetary continuum radiations or other planetary radio emission phenomena.

Another terrestrial auroral radio emission, also detectable at ground level, is Medium Frequency Burst (MFB), first reported by Weatherwax et al. [1994] although it was possibly observed previously but not clearly identified [see discussion in LaBelle and Treumann, 2002, pp. 406-407]. MFB is the least understood of the terrestrial auroral emissions, but like auroral roar, it is a nearby, accessible planetary radiation source, and it is associated with polar substorms, one of the most significant energy release phenomena in the terrestrial magnetosphere. LaBelle and Treumann [2002] provided a thorough review of MFB, but recent developments in both experiment and theory, reviewed below, have made significant progress toward understanding this emission which, like auroral roar, may turn out to share characteristics with less accessible emissions such as solar radio bursts.

\section{Review of Recent Observational Work}

Figure 1 shows four examples of MFB recorded at four different stations using the Dartmouth College LF/MF/HF swept frequency receiver which has a time resolution of 1 $\mathrm{s}$ and frequency resolution of $10 \mathrm{kHz}$. With these resolutions, MFB appears as a cluster of impulsive emissions spanning a 500-2000 kHz range between 1400 and $4500 \mathrm{kHz}$. Two different experiments have shown that MFB is left-hand polarized, implying that it propagates in the LO-mode in the ionosphere [Shepherd et al., 1997; Sato et al., 2008].

Much evidence suggests that MFB coincides with the onset of polar substorms, often together with other auroral radio emissions such as auroral roar and hiss [Weatherwax et al., 1994; LaBelle et al., 1994]. The correlation with LF impulsive auroral hiss is particularly striking; for example, Figure 1a shows a sequence of seven clusters of MFB emissions, each coincident with a cluster of impulsive auroral hiss at frequencies below $500 \mathrm{kHz}$. Figures 1b-c show similar good correlations between MFB and hiss. Several papers have shown that both MFB and impulsive hiss coincide with the prompt onset of auroral absorption registered by local riometers and with deflections of components of the magnetic field measured with local magnetometers, both of which are signatures of substorm onset [LaBelle et al., 1994, 1997, 2005]. In Figures 1a and 1c, MFB coincides with prompt attenuation of broadcast band signals at 550-1700 kHz, another indicator of substorm onset. By measuring the direction of arrival of MFB combined with ray tracing analysis, Bunch et al. [2008] showed that MFB originates in active auroral arcs during the expansion phase of one of the THEMIS study substorms. Sato et al. [2008] studied two MFB events in detail: in one case, substorm onset started about one minute after the $\mathrm{MFB}$, and in the other, enhanced activity commenced toward the end of the MFB; both were associated with $>1100 \mathrm{nT}$ peaks in the AE index. Its prompt, well-defined onset combined with occurrence at or just before the breakup phase of polar substorms makes MFB potentially useful for location and timing of substorm onsets. 

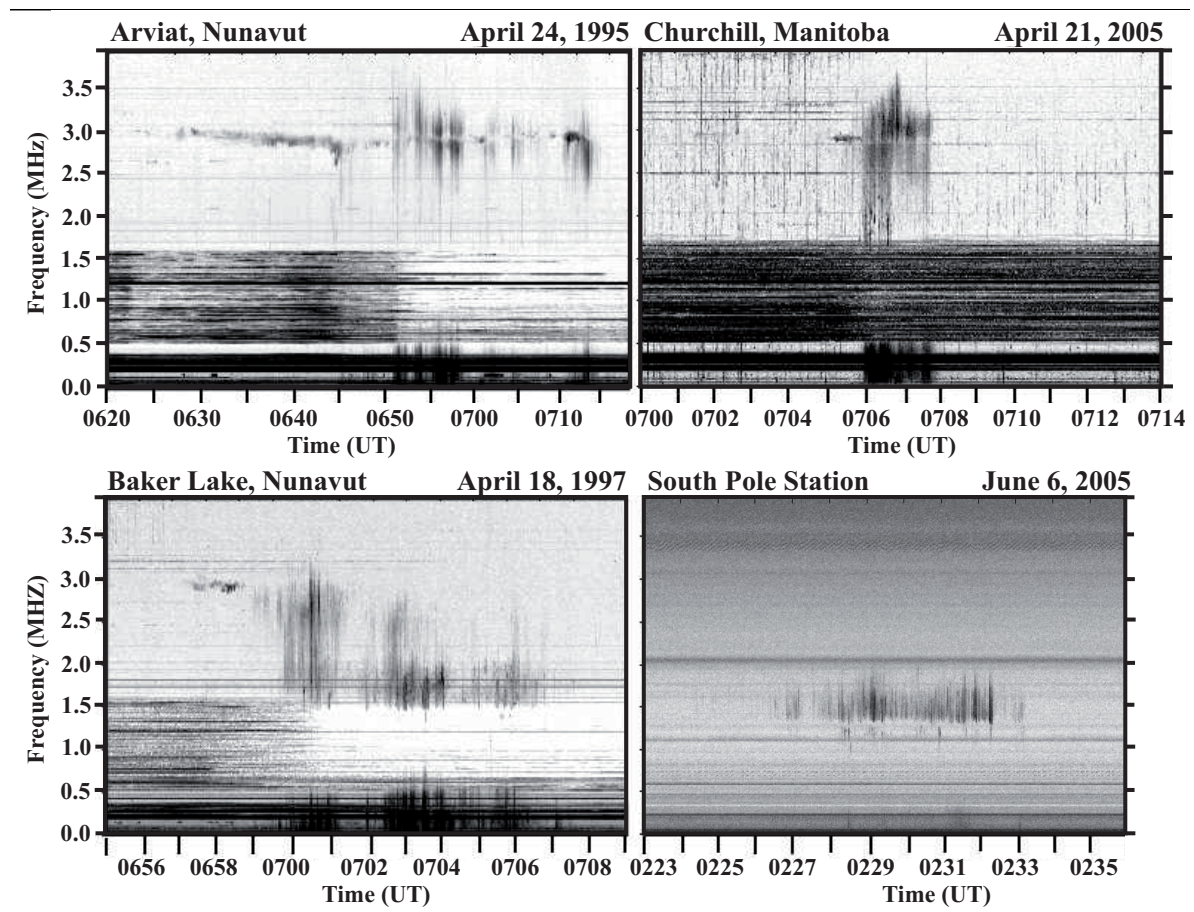

Figure 1: Examples of auroral radio emissions, including MFB, recorded by Dartmouth College radio receivers at various observatories: (a) Arviat, Nunavut (71.3 invariant), showing an extraordinarily long lasting sequence of MFB (0652-0714 UT), displaying the characteristic null near $3 \mathrm{MHz}$ and correlated with auroral hiss emissions below $500 \mathrm{kHz}$; (b) Churchill, Manitoba (69.4 invariant) showing the null near $3 \mathrm{MHz}$ and correlation with hiss; (c) Baker Lake, Nunavut (74.2 $2^{\circ}$ invariant) showing a distinct lower frequency boundary near the ionospheric electron gyrofrequency $(1500 \mathrm{kHz})$ and occasional emissions at lower frequencies below a gap; South Pole (74. $4^{\circ}$ invariant) also showing the lower cutoff, frequency gap, and emission below the gyrofrequency.

The duration of MFB at ground level ranges from a few seconds to half an hour, but is typically a few minutes. The short duration is caused by ionospheric propagation conditions. Coincident radio and optical all-sky camera images show that the extinction of MFB occurs when the aurora moves overhead of the station, presumably due to absorption in the enhanced low-altitude ionization created by the auroral electron beam [Bunch et al., 2010]. The absence of MFB during sunlight also points to the role of ionospheric absorption in determining MFB occurrence. The occurrence rate of MFB depends somewhat on auroral activity; Weatherwax et al. [1993] report events on 28 out of 180 nights, LaBelle et al. [1997] report occurrence during 5-10 percent of one-hour intervals studied, and a recent survey of radio data from Churchill, Manitoba, yields about 850 events over 15 winter seasons. Occurrence rates peak in the premidnight MLT sector [Fig. 3 of 
LaBelle et al., 1997]. The important role of ionospheric propagation conditions probably explains why not every substorm onset is associated with detectable MFB, but virtually every MFB is associated with a substorm onset.

LaBelle et al. [2005] use observations from a meridional chain of receivers spanning 69$79^{\circ}$ invariant latitude to show that as the substorm expands poleward, MFB appears at successively more poleward receivers. More recently, Bunch et al. [2009] improved on this result by making direction of arrival (DOA) measurements of MFB as the substorm expansion phase developed. In this case, the receiver at Toolik Lake, Alaska, was ideally situated poleward of the northward-expanding substorm auroral arcs, providing a good propagation path from the arcs to the receiver through the undisturbed polar cap ionosphere. As the most poleward arc expanded northward, the DOA of the MFB emission shifted northward. In this experiment, the source of the MFB appeared to be the most poleward expanding substorm arc.

Bunch et al. [2009] made another interesting recent observation concerning the DOA of auroral MFB: in a number of examples recorded at Toolik Lake, Alaska, higher MFB frequency components had higher elevation angles of arrival than did lower frequency components.

LaBelle and Treumann [2002, pp. 404-405] review in detail the range of intensities of MFB. The field strength averaged over 1 -s time intervals and $10-\mathrm{kHz}$ frequency intervals is typically $50-100 \mathrm{mV} / \mathrm{m}$. An example shown by Sato et al. [2008] (their Figure 8) appears to fall in this range, for example. The peak power could be up to a factor ten higher. These signal strengths imply average (peak) source powers of $100 \mathrm{~W}(1000 \mathrm{~W})$ [LaBelle et al., 1997]. These power levels are comparable to those for auroral hiss and somewhat greater than those for auroral roar, though the longer duration of auroral roar implies that similar amounts of energy are involved in each of these emissions. The power level of any of these is far smaller than that emitted in Auroral Kilometric Radiation.

Two experiments suggest a relationship between the upper frequency bound of MFB and the maximum electron density in the overlying ionosphere. The maximum frequency of $\mathrm{MFB}$ recorded at Sondrestrom, Greenland, jumped from $\sim 2 \mathrm{MHz}$ to $\sim 3 \mathrm{MHz}$ at the same time that the maximum plasma frequency in the ionosphere, deduced from incoherent scatter radar data, jumped between similar values [LaBelle et al., 1997, Fig. 10]. Less directly, LaBelle et al. [2005] measured the frequency ranges of all MFB events recorded during an entire year at South Pole Station and found that the upper frequency bound increased when solar zenith angle decreased, as expected if higher electron density controlled by photoionization were associated with higher MFB upper frequency bound.

Figure 1 shows examples of two types of frequency structure seen in MFB. Often, when the emission spans $\approx 3 \mathrm{MHz}$, which is approximately twice the ionospheric electron gyrofrequency, there is a null or decrease in power spectral density there. Weatherwax et al. [1993] show statistical evidence for this null; often MFB emissions span the frequency range between the ionospheric gyrofrequency and its harmonic, or between the gyro-harmonics, without spanning the gyrofrequency. Figures 1a and 1b show examples of the null which occurs when MFB spans the gyroharmonic. Figures 1c and 1d show a second feature which occurs when MFB extends below about $1400 \mathrm{kHz}$, approximately 
the ionospheric electron gyrofrequency. In these cases, there is often a sharply defined lower cutoff of the MFB with a gap below the cutoff, and the MFB power spectral density picks up again well below the cutoff. LaBelle and Treumann [2002] suggest that the MFB below the cutoff is whistler mode, whereas that above the cutoff is LO-mode, but no polarization measurements exist to confirm that.

High-speed narrowband measurements of MFB suggested the presence of substructure [LaBelle et al., 1997], and Bunch and LaBelle [2009] reported the first fully-sampled waveform measurements of MFB which revealed details of the characteristic substructures. These consist of bursty emissions lasting tens of milliseconds with a sharply defined leading edge. The leading edge has a "nose frequency" with rapid dispersion at frequencies above the nose and gradual dispersion at frequencies below the nose, leading to an "invertedseven" shape of the leading edge. Figure 2, reprinted from Bunch and LaBelle [2009], shows two examples. MFB also includes a faint background continuum which may consist of multiple overlapping weaker substructures. These substructures provide an important test of MFB generation mechanisms.

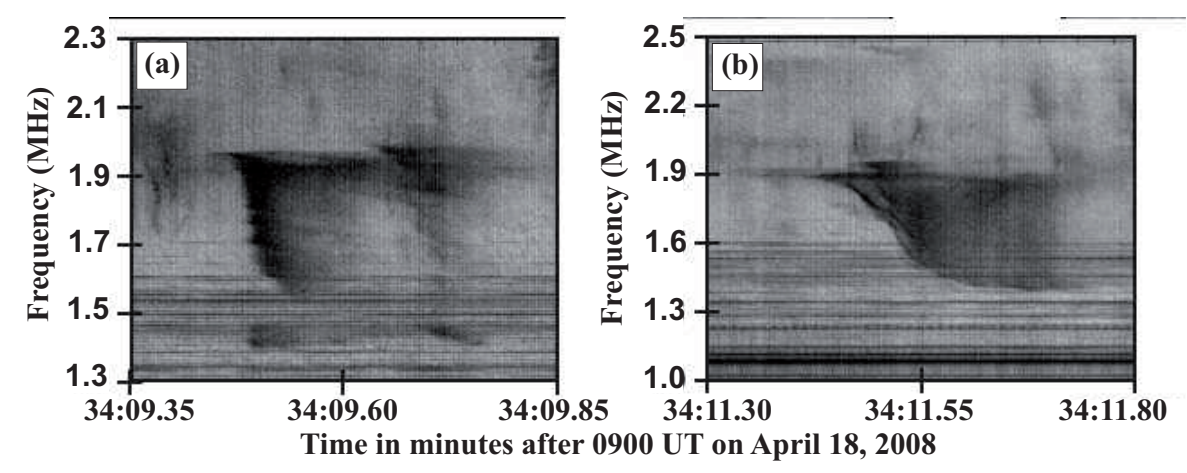

Figure 2: Two examples of MFB substructure recorded at Toolik Lake, Alaska, and reprinted from Bunch and LaBelle [2009], showing the characteristic "inverted-seven" leading edge feature described in the text. (Reprinted with permission of the American Geophysical Union.

\section{Review of Recent Theoretical Work}

Three mechanisms have been put forth in the literature to explain MFB emissions; all involve mode conversion of electrostatic waves excited by nonthermal electron distributions in the auroral ionosphere. LaBelle et al. [1997] proposed that Langmuir or upper hybrid waves, excited over a range of altitudes on the bottomside of the F-region ionosphere, mode convert to L-mode EM waves and propagate to the ground. LaBelle [2011] proposes a variation on this, whereby Langmuir waves excited over a range of altitudes on the topside of the F-region ionosphere linearly transform to L-mode EM waves and propagate to the ground. Sotnikov et al. [1996] propose that broadband electron acoustic waves generated by a gyrating electron beam, possibly at one location in the ionosphere, 
convert to L-mode EM waves and propagate to the ground. Recently, Bunch et al. [2011] evaluate these mechanisms and find them plausible from the point of view of resonance with auroral electron beams. Figure 3 shows a schematic of each mechanism.

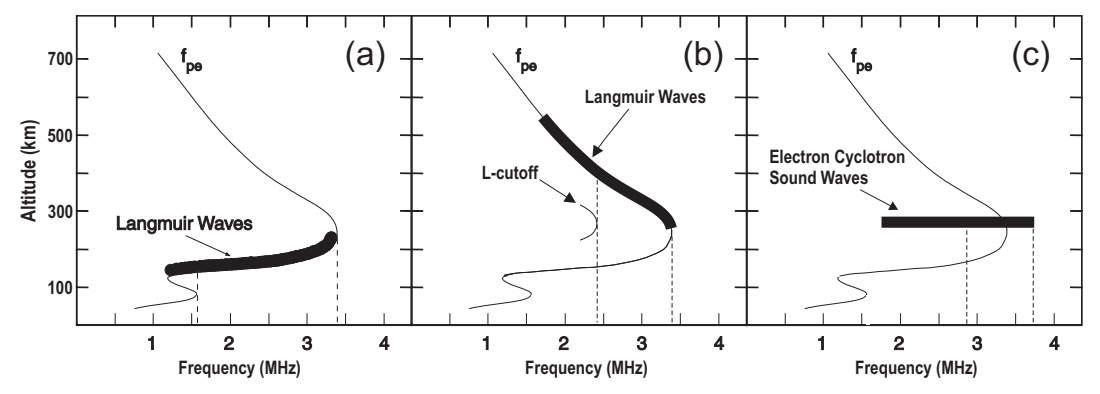

Figure 3: Illustrations of three mechanisms proposed to explain generation of MFB: (a) mode conversion of Langmuir waves generated over a range of altitudes (and plasma frequencies) in the bottomside ionosphere; (b) mode conversion of Langmuir waves generated over a range of altitudes in the topside ionosphere; and (c) mode conversion of electron cyclotron sound waves generated at a single source in the ionosphere, near the F-peak where the electron density is relatively large.

The auroral ionosphere includes at least three electron populations: the background thermal population, the auroral electron beam, and warm secondary electrons. Such a plasma can support two additional normal modes called the electron acoustic (EA) and electron cyclotron sound (ECS) waves. The electron acoustic mode has been investigated as a possible source of cusp auroral hiss [Tokar and Gary, 1984; Mace and Hellberg, 1993]; the electron cyclotron sound wave has been investigated as a candidate source mechanism for broadband waves generated in active experiments [Sotnikov et al., 1995] as well as natural emissions [Mace and Hellberg, 1993]. Sotnikov et al. [1996] propose that generation of ECS by a gyrating electron beam might be a source mechanism for MFB. The advantage of this mechanism is that it generates a broad frequency range from a single source, so there is no need to postulate beam persistence over a range of altitudes. Bunch et al. [2011], using the WHAMP plasma wave dispersion code, replicated some of the results of Mace and Hellberg [1993] concerning ECS and then explored the conditions for cyclotron or Landau resonances between this mode and the auroral electrons. Despite some limitations of their computational technique, their results suggest that relatively high electron densities $\left(\omega_{p e}\right.$ several times $\left.\omega_{c e}\right)$ and warm secondary populations (of order $100 \mathrm{eV}$ ) are required in order for the mode to be excited over a sufficiently broad range of frequencies (up to $2 f_{c e}$ ) to explain MFB. Such conditions are reasonable in active aurora arcs. Of course, the ECS waves are electrostatic and require conversion to LO mode in order to propagate to ground level. Thus far, only limited computations have been done on the ECS mode applied to the aurora, but the results warrant a more thorough investigation of the dispersion of the mode for realistic plasma parameters, its growth or damping in 
the presence of auroral electrons, and relevant mode conversion mechanisms.

Spacecraft measurements have long shown that the auroral electron beam generates in the ionosphere Langmuir waves with amplitudes that often reach hundreds of $\mathrm{mV} / \mathrm{m}$ [e.g., McFadden et al., 1986; Boehm, 1987] Excitation of Langmuir or upper hybrid waves over a range of altitudes on the bottomside ionosphere, followed by conversion to LO-mode electromagnetic waves, would easily explain both the broad frequency range and the propagation to ground level of MFB emissions [LaBelle et al., 1997]. This mechanism predicts that the maximum MFB frequency should be the plasma or upper hybrid frequency at the F-peak, and the minimum frequency would correspond either to the altitude at which the electron beam no longer excites Langmuir waves or to the maximum L-cutoff in an underlying E-layer. For all recorded MFB's, maximum frequencies lie in the range 1.5-4.5 $\mathrm{MHz}$, which plausibly lies below maximum plasma frequencies in the auroral F-region. Furthermore, as discussed above, there is evidence that the maximum frequency of MFB is related to the maximum plasma frequency in the overlying ionosphere. Direction finding measurements suggesting that high-frequency MFB components have higher arrival elevation angles than low-frequencies are also consistent with generation at the plasma frequency on the bottomside, where high plasma frequencies lie at higher altitudes than low plasma frequencies. For either the Langmuir or upper hybrid modes, mode conversion to LO-mode electromagnetic waves is possible through a radio window, as probably occurs for auroral roar emissions [e.g., Yoon et al., 1998]. A disadvantage of the bottomside source is that it requires the auroral electron beam to persist over a range of low altitudes.

LaBelle [2011] puts forth a variation on this mechanism in which the causative Langmuir waves are in the topside ionsophere. In this scenario, the causative Langmuir waves refract onto the Z-mode branch as they propagate downward into increasing electron density, and then as the propagate into decreasing electron density on the bottomside ionosphere, some fraction which refract to have parallel wave vector linearly convert to the L-mode and can reach ground level. (Due to a typographical error, the words "parallel" and "perpendicular" are switched in the description given by LaBelle [2011].) This mechanism also requires excitation of Langmuir waves over a range of altitudes, but at considerably higher altitudes. A topside source also implies predictions of the MFB frequency range: the maximum MFB frequency must lie below the maximum plasma frequency and the minimum frequency must exceed the maximum L-cutoff frequency at the F-peak; for the six examples of MFB fine structure shown by Bunch et al. [2009], assuming the maximum observed frequency is $f_{p e}$ implies that the minimum observed frequency exceeds the L-cutoff. Another advantage of this mechanism is that linear transformation of the Langmuir waves into LO-modes occurs naturally because the waves generated by the downgoing electron beam propagate downward into increasing densities where those with parallel wavenumbers encounter the radio window. In fact, consideration of the time delays due to electron motion and wave propagation explains the "backwards-seven" features of the leading edges of the MFB fine structure [LaBelle, 2011]. The fit between the predicted dispersion and the fine structure measurements works only for relatively low energy electron beams $(<1 \mathrm{keV})$. Interestingly, this result together with the association of MFB with the poleward expanding substorm auroral arc [Bunch et al., 2009], supports an increasing body of evidence and theory that these poleward expanding arcs are dominated by Alfvénically accelerated electrons [e.g., Mende et al., 2003ab; Semeter 
et al., 2008; Sakaguchi et al., 2009]. Disadvantages of this mechanism include: it appears inconsistent with the Bunch et al. [2009] measurements of the frequency dependence of MFB elevation angles, at least for simple a slab model ionosphere, though propagation calculations including horizontal density structure may change that conclusion; and the collisionless damping of the Langmuir waves requires careful consideration since they must propagate a few tens of meters along the field in order to undergo linear transformation to LO mode. The collisional damping distance is only a few meters at the source of the waves but grows to be hundreds of meters near the mode conversion point, so full-wave calculations may be required to assess the wave attenuation.

The mode conversion efficiency required by this mechanism depends on the source power per bandwidth inferred from the measured MFB amplitudes compared to that of the causative electrostatic Langmuir waves. MFB emissions have broadband amplitudes 50$100 \mu \mathrm{V} / \mathrm{m}$ and bandwidth $B W_{E M} \sim 1 \mathrm{MHz}$. Since they are free space waves, their energy density is given by $\epsilon_{0} E^{2} c / 2\left(\right.$ in $\left.\mathrm{W} / \mathrm{m}^{2}\right)$. Dividing by bandwidth and multiplying by area illuminated ( $=\Delta \Omega_{E M} R_{E M}^{2}$, where $R_{E M}$ is the distance of the observation from the source and $\Delta \Omega_{E M}$ is the solid angle illuminated by the source) yields the emitted power per Hertz in Watts:

$$
P_{E M}=\frac{\epsilon_{0} E_{E M}^{2}}{2} c \frac{1}{B W_{E M}} \Delta \Omega_{E M} R_{E M}^{2}
$$

The source distance for MFB is approximately $300 \mathrm{~km}$, and the solid angle must be substantial, of order one steradian, since ground stations hundreds of kilometers apart sometimes observe the same MFB emissions. Equation (1) neglects absorption or scattering of electromagnetic energy between the source and the ground level, an effect which would increase the required source power somewhat.

The Langmuir wave energy density is given by $\epsilon_{0} E^{2}$, where the factor of two accounts for the additional energy stored in the electron motion, providing the polarization of the plasma, as discussed on pages 216-219 of Baumjohann and Treumann [1996]. The equivalent power per bandwidth for the Langmuir waves must take into account that they are being pumped by the unstable auroral electron beam:

$$
P_{L}=2 \frac{\epsilon_{0} E_{L}^{2}}{2} V \frac{1}{B W_{L}} \gamma_{L}
$$

where $B W_{L}$ is the Langmuir wave bandwidth, $V$ is the source volume, and $\gamma_{L}$ is the growth rate due to the unstable auroral electron beam. Rocket observations show that auroral Langmuir waves at the local plasma frequency $(\sim 1 \mathrm{MHz})$ have electric field amplitudes $E_{L}$ up to $1 \mathrm{~V} / \mathrm{m}$ [e.g., Boehm, 1987] and bandwidths $\left(B W_{L}\right)$ less than $1 \mathrm{kHz}$. (In fact, one example studied by Kletzing et al. [2005] has considerably narrower bandwidth.) Taking the source size to be approximately $30 \mathrm{~m}$ implies $V \sim 10^{4} \mathrm{~m}^{3}$. The growth rate may be estimated from the "gentle beam" analysis on pages 70-73 of Treumann and Baumjohann [1997]:

$$
\gamma_{L}=\left(\frac{\pi}{2 \mathrm{e}}\right)^{1 / 2} \frac{N_{b}}{N_{0}}\left(\frac{v_{b} \cos \theta}{v_{t h}}\right)^{2} \omega_{p e}
$$

where e is the base of the natural logarithms, $N_{b}$ and $N_{0}$ are the beam and background electron densities, $v_{b}$ and $v_{t h}$ are the beam and thermal electron temperatures, $\theta$ is the 
angle between the k-vector and the ambient magnetic field, and $\omega_{p e}=2 \pi f_{p e}$ is the plasma frequency. For typical auroral electron beams, $N_{b} / N_{0} \sim 10^{-4}$ and $\left(v_{b} / v_{t h}\right)^{2} \sim 10^{4}$. Taking $\theta \sim 0$ and $\omega_{p e} \sim 10^{7}$ therefore implies $\gamma_{L} \sim 10^{7} \mathrm{~s}^{-1}$

Inserting measured electric fields and bandwidths, as well as the results of equation (3), into (1) and (2) leads to $P_{E M} / P_{L} \sim 1.5 \times 10^{-3}$. As mentioned above, absorption effects could reduce this ratio somewhat. The largest uncertainty in the calculation is probably the source volume which is not measured. If the source were $10 \mathrm{~m}$ rather than $30 \mathrm{~m}$, the ratio would be reduced by an order of magnitude. It is unlikely that the source is much smaller than $10 \mathrm{~m}$ since that is approximately the wavelength of the causative Langmuir waves (assuming $1 \mathrm{MHz}$ frequency and resonance with $1 \mathrm{keV}$ electron beam). Therefore, these calculations suggest that only a relatively small portion of the Langmuir wave power, perhaps less than one percent, needs to convert to EM in order to explain the observations.

Incidentally, a similar conclusion arises from considering the relative energy densities of the two modes in the source region, which can also be estimated from the measured MFB amplitudes and in-situ rocket measurements of auroral Langmuir waves. For the free-space waves, the energy density is given by $\epsilon_{0} E^{2} / 2$; the energy density in the source depends, however, on the distance from the source. Assuming a source size of radius $30 \mathrm{~m}$ and source distance of $300 \mathrm{~km}$ implies the radiation electric field in the source is enhanced by a factor $10^{4}$ over the observed ground-level value (energy density increased by factor $10^{8}$ ). This calculation neglects propagation effects of absorption and scattering which would require higher energy density in the source region. The Langmuir wave energy density is given by $\epsilon_{0} E^{2}$, where the factor of two accounts for the additional energy stored in the electron motion, providing the polarization of the plasma, as mentioned above. Plugging in the measured electric fields and adjusting for the bandwidths, one obtains $\sim 5 \times 10^{-18} \mathrm{~J} / \mathrm{m}^{3} \mathrm{~Hz}$ for the energy density per bandwidth of the EM waves $\sim 9 \times 10^{-15} \mathrm{~J} / \mathrm{m}^{3} \mathrm{~Hz}$ for that of the causative Langmuir waves. Hence the Langmuir wave energy density per bandwidth in the source exceeds that of the mode converted radiation by a factor of $\sim 2000$. If the source were $10 \mathrm{~m}$ radius instead of $30 \mathrm{~m}$, the ratio would reduce to 200. As mentioned above, absorption effects could reduce this ratio somewhat. However, this calculation also suggests that only a small portion of the Langmuir wave energy, perhaps less than one percent, needs to convert to EM in order to explain the observations.

\section{Conclusions}

The MFB emission remains the least understood of the terrestrial auroral radio emissions, although recent measurements of the emission fine structure [Bunch et al., 2009] provide unprecedented opportunities for quantitative testing of proposed generation mechanisms. Linear transformation of downgoing Langmuir waves excited over a range of altitudes in the topside ionosphere by relatively low-energy downgoing electron beams appears quantitatively consistent with MFB fine structure dispersion [LaBelle, 2011], although beam persistence, Langmuir wave collisionless damping, and elevation angle as a function of frequency at ground level need to be evaluated. Ground-level measurements of MFB and rocket measurements of auroral Langmuir waves suggest that the Langmuir wave 
power and energy density per bandwidth exceed those of the EM waves in the source region by 2-3 orders of magnitude. More theoretical work on electron cyclotron sound waves is warranted to test whether these provide an alternative explanation for MFB fine structure and intensity, without requiring wave generation over a range of altitudes. The prevalence of mode conversion radiation in a broad range of space environments, including planetary ionospheres and magnetospheres as well as the solar wind, makes a compelling argument for striving to understand those examples generated in the terrestrial ionosphere and observable at ground level.

Acknowledgements. The author thanks the referee for his or her critical comments, R.A. Treumann for many helpful discussions, and A.T. Weatherwax, S.G. Shepherd, N. Bunch, and M. Broughton for their work on the MF Burst emission over the years. This work was supported by National Science Foundation grants ATM-0753198, NSF-0838745, and ANT-0840669 to Dartmouth College.

\section{References}

Baumjohann, W., and R.A. Treumann, Basic Space Plasma Physics, Imperial College Press, London, 1996.

Boehm, M.H., Waves and static electric fields in the auroral acceleration region, Ph.D. thesis, Dept. of Physics, Univ. of California at Berkeley, 1987.

Bunch, N.L., and J. LaBelle, Fully resolved observations of auroral medium frequency burst radio emissions, Geophys. Res. Lett., 36, L15104, doi:10.1029/2009GL038513, 2009 .

Bunch, N. L., J. LaBelle, A. T. Weatherwax, J. M. Hughes, Auroral Medium Frequency Burst radio emission associated with the March 23, 2007, THEMIS study substorm, J. Geophys. Res., 113, A00C08, doi:10.1029/2008JA013503, 2008.

Bunch, N. L., J. LaBelle, A. T. Weatherwax, J. M. Hughes, Experimental Tests of the Generation Mechanism of Auroral Medium Frequency Burst Radio Emissions, J. Geophys. Res., 114, A09302, doi:10.1029/2008JA013993, 2009.

Bunch, N.L., J. LaBelle, P. H. Yoon, and A. T. Weatherwax, Theoretical Constraints on the Generation Mechanism of Auroral Medium Frequency Burst Radio Emissions, J. Geophys. Res., 116, A01315, doi:10.1029/2010JA015951, 2011.

Kellogg, P. J., and S. J. Monson, Radio emissions from the aurora, Geophys. Res. Lett., 6, 4, 297, 1979 .

Kletzing, C.A., S.R. Bounds, J. LaBelle, and M. Samara, Observation of the reactive component of Langmuir wave phase-bunched electrons, Geophys. Res. Lett., 23, L05106, doi:10.1029/2004GL021175, 2005.

LaBelle, J., An Explanation for the Fine Structure of MF Burst Emissions, Geophys. Res. Lett., 38, L03105, doi:10.1029/2010GL046218, 2011. 
LaBelle, J., and A. T. Weatherwax, Ground-based observations of LF/MF/HF radio waves of auroral origin, in Proceedings of 1992 Cambridge Workshop in Geoplasma Physics, edited by T. Chang, Scientific Publishers, Cambridge, Mass., 223, 1993.

LaBelle, J., and R. A. Treumann, Auroral Radio Emissions, 1. Hisses, Roars, and Bursts, Space Sci. Rev., 101, 295-440, 2002.

LaBelle, J., A. T. Weatherwax, M. L. Trimpi, R. Brittain, R. D. Hunsucker, and J. V. Olson, The spectrum of LF/MF/HF radio noise at ground level during geomagnetic substorms, Geophys. Res. Lett., 21, 2749, 1994.

LaBelle, J., S. G. Shepherd, and M. L. Trimpi, Observations of auroral medium frequency burst emissions, J. Geophys. Res., 102, 22221, 1997.

LaBelle, J., A. T. Weatherwax, M. Tantiwiwat, E. Jackson, and J. Linder, Statistical study of medium-frequency burst emissions observed at South Pole Station and at multiple Canadian Observatories, J. Geophys. Res., 110, No. A2, A02305, doi:10.1029/2004JA010608, 2005.

Mace, R. L., and M. A. Hellberg, Electron acoustic and cyclotron sound instabilities driven by field aligned hot electron streaming, J. Geophys. Res., 98, 5881-5891, 1993.

McFadden, J. P., C. W. Carlson, and M. H. Boehm, High frequency waves generated by auroral electrons, J. Geophys. Res., 91, 12079, 1986.

Melrose, D. B., The brightness temperatures of solar type III bursts, Solar Phys., 120, 369-381, 1989.

Melrose, D. B., and M. V. Goldman, Microstructures in type III events in the solar wind, Solar Phys., 107, 329, 1987.

Mende, S. B., C.W. Carlson, H. U. Frey, T. J. Immel, and J.-C. Gérard, IMAGE FUV and in situ FAST particle observations of substorm aurorae, J. Geophys. Res., 108, A4, 8010, doi:10.1029/2002JA009413, 2003a.

Mende, S. B., C.W. Carlson, H. U. Frey, L. M. Peticolas, and N. Ostgaard, FAST and IMAGE-FUV observations of a substorm onset, J. Geophys. Res., 108, A9, 1344, doi:10.1029/2002JA009787, 2003b.

Sakaguchi, K., K. Shiokawa, and E. Donovan, Azimuthal structures of ray auroras at the beginning of auroral substorms, Geophys. Res. Lett., 36, L23106, doi:10.1029/2009GL041252, 2009.

Samara, M., J. LaBelle, C.A. Kletzing, and S. R. Bounds, Electrostatic upper hybrid waves where the upper hybrid frequency matches the electron cyclotron harmonic in the auroral ionosphere, Geophys. Res. Lett., 31, L22804, doi:10.1029/2004GL021043, 2004.

Sato, Y., T. Ono, M. Iizima, A. Kumamoto, N. Sato, A. Kadokura, and H. Miyaoka, Auroral radio emission and absorption of medium frequency radio waves observed in Iceland, Earth Plan. Space, 60, 207-217, 2008. 
Semeter, J., M. Zettergren, M. Diaz, and S. Mende, Wave dispersion and the discrete aurora: New constraints derived from high-speed imagery, J. Geophys. Res., 113, A12208, doi:10.1029/2008JA013122, 2008.

Shepherd, S. G., J. LaBelle, and M. L. Trimpi, The polarization of auroral roar emissions, Geophys. Res. Lett., 24, 3161, 1997.

Sotnikov, V., D. Schriver, M. Ashour-Abdalla, J. Erstmeyer, and N. Myers, Excitation of electron acoustic waves by a gyrating electron beam, J. Geophys. Res., 100, 19765, 1995.

Sotnikov, V., D. Schriver, M. Ashour-Abdalla, and J. LaBelle, Generation of auroral radio waves by a gyrating electron beam, EOS Trans. Am. Geophys. Union, 77, F544, 1996.

Tokar, R. L., and S.P. Gary, Electrostatic hiss and the beam driven electron acoustic instability in the dayside polar cusp, Geophys. Res. Lett., 11, 1180, 1984.

Treumann, R., and W. Baumjohann, Advanced Space Plasma Physics, Imperial College Press, 1997.

Weatherwax, A. T., J. LaBelle, M. L. Trimpi, and R. Brittain, Ground based observations of radio emissions near $2 f_{c e}$ and $3 f_{c e}$ in the auroral zone, Geophys. Res. Lett., 20, $1447,1993$.

Weatherwax, A.T., J. LaBelle, M.L. Trimpi, A new type of auroral radio emission at 1.4-3.7 MHz observed from the ground, Geophys. Res. Lett., 21, 2753, 1994.

Yoon, P. H., A. T. Weatherwax, T. J. Rosenberg, and J. LaBelle, Lower ionospheric cyclotron maser theory: A possible source of $2 f_{c e}$ and $3 f_{c e}$ auroral radio emissions, $J$. Geophys. Res., 101, 27015, 1996.

Yoon, P. H., A. T. Weatherwax, T. J. Rosenberg, and J. LaBelle, and S. G. Shepherd, Propagation of Medium Frequency (1-4 MHz) Auroral Radio Waves to the Ground via the Z Mode Radio Window, J. Geophys. Res., 103, 29267, 1998. 